\title{
Changer les mentalités pour une culture entrepreneuriale : exemples de pratiques de sensibilisation réussies au Québec
}

\author{
Hela Ben Ayed \\ Carrefour jeunesse emploi Saint-Laurent
}

\section{Introduction}

$\Gamma$ a situation de l'entrepreneuriat au Québec est aujourd'hui semblable à plusieurs pays : une population qui vieillit et un départ massif d'entrepreneurs en retraite qui entraînent une baisse du nombre des PME et un taux de création d'entreprises de plus en plus réduit. Nous comprenons que la situation est dangereuse si l'on considère que les PME constituent un moteur de croissance économique et un facteur déterminant de richesse d'un pays. Comme le mentionne Fortin ${ }^{1}$, la création d'entreprises est la principale activité permettant de combattre la pauvreté et d'assurer le développement d'une société. Face à une telle situation, chaque pays répond à cette problématique à sa manière en adoptant des solutions à court ou à long terme adaptées à son contexte. Le Québec a choisi une stratégie globale à long ter-

me qui se traduit par un changement de culture. Le Québec s'est lancé le défi de changer les mentalités qui négligeaient autrefois et même dénigraient parfois l'entrepreneuriat à de nouvelles mentalités qui favorisent et encouragent l'esprit entrepreneurial.

Plusieurs initiatives ont été mises en avant en matière de sensibilisation à l'entrepreneuriat. Ces efforts commencent à donner fruit et sont parfaitement transposables, notamment aux pays qui ont la problématique de la relève. Le gouvernement québécois a compris qu'on doit miser sur les plus jeunes pour continuer à bâtir des entreprises. En posant comme postulat que l'esprit entrepreneurial se cultive à l'école dès le plus jeune âge, des initiatives ont été mises en place partout au Québec. C'est à l'école qu'on peut amener les jeunes à s'éveiller au plaisir d'entreprendre. Les jeunes doivent donc être sensibilisés aux valeurs entre- preneuriales le plus tôt possible afin de leur donner la piqûre entrepreneuriale et de faire de l'entrepreneuriat un choix de carrière désirable.

À cet effet, le Défi de l'entrepreneuriat jeunesse ${ }^{2}$, lancé par le gouvernement québécois en février 2004, vise à faire face à ce contexte de la relève. L'objectif de ce plan d'action triennal de $21 \mathrm{M} \$$ est de développer la culture entrepreneuriale et les valeurs qu'elle sous-tend : l'autonomie, la créativité, la confiance en soi, le leadership, la responsabilité et la solidarité auprès des jeunes. Le premier axe sur lequel repose ce défi est de reconnaittre l'école comme un élément essentiel au renforcement de la culture entrepreneuriale chez les jeunes. Cet objectif rejoint l'esprit du projet éducatif du ministère de l'Éducation. Ce dernier cherche à amener les élèves à entreprendre des projets et à réaliser des actions orientées vers la connaissance et la réalisation de soi ainsi que l'engagement dans la société. Qu'il s'agisse de programmes scolaires ou parascolaires, les établissements d'enseignement primaire, secondaire, collégial et universitaire apparaissent comme des lieux privilégiés pour la promotion de la culture entrepreneuriale.

De plus, dans le cadre du Défi de l'entrepreneuriat jeunesse, 75 agents de sensibilisation à l'entrepreneuriat jeunesse ont été mandatés par le gouvernement pour tendre à changer les mentalités vers une culture entrepreneuriale. Leur rôle est d'allumer la flamme entrepreneuriale chez les jeunes et ce, en rencontrant les professeurs des différents niveaux scolaires et universitaires pour les informer et les accompagner dans la mise en place des projets entrepreneuriaux. Ce sont des agents de changement qui, par leur discours de persuasion, tentent à être des agents multiplicateurs 
pour le développement d'une véritable culture entrepreneuriale. Ces agents font la promotion non seulement auprès du milieu scolaire, mais aussi auprès des parents, des élus municipaux et de la communauté en général.

Il est temps de changer de paradigmes. L'entrepreneuriat, qui avait une connotation négative dans le passé, est aujourd'hui de plus en plus valorisé. Tout le monde est mobilisé par cette nouvelle culture. Il s'agit d'une approche multi acteurs. La synergie qui se développe entre les intervenants en éducation, d'une part, et la communauté autour de l'école, d'autre part, est essentielle pour permettre aux étudiants de mettre en avant leur potentiel et de réaliser leurs rêves. Nous présenterons dans cet article l'approche d'une nouvelle culture entrepreneuriale, puis nous explorerons les facteurs clés de succès de la communication autour du changement culturel, et enfin nous présenterons des exemples de pratiques de sensibilisation à l'entrepreneuriat dans le milieu scolaire et universitaire au Québec.

\section{La culture entrepreneuriale : un changement de mentalités}

La culture est un concept complexe. C'est un ensemble de connaissances, valeurs, croyances et références que partagent les individus d'une même société et qui influencent les comportements. Ainsi, la culture englobe la carte mentale collective qui est beaucoup plus puissante que le cadre de référence individuel. Il devient donc très difficile de changer la culture en peu de temps. Par ailleurs, la culture est parfaitement évolutive lorsqu'on se donne les moyens pour agir. Changer la culture risque de prendre beaucoup de temps. En effet, il ne s'agit pas d'un changement radical mais plutôt incrémental puisqu'il s'agit de toucher les valeurs enracinées et intégrées au sein d'une population depuis plusieurs générations.

Si l'on reprend la définition de Fortin de la culture entrepreneuriale, on retrouve trois éléments : 1) des connaissances qu'un entrepreneur ou un employé aurait besoin pour relever des défis, 2) des attitudes et des valeurs (créativité, sens de la responsabilité, autonomie, confiance en soi, solidarité, leadership, etc.), 3) des compétences de savoir-faire, savoir-être et savoir-agir. Pour ce qui est des connaissances, il est généralement facile de les développer à travers les formations privées ou publiques, les programmes instaurés par le ministère de l'Éducation, etc. Le Québec est bien avancé dans ce domaine, tout comme la France et plusieurs autres pays touchés par la problématique de la relève. Par contre, pour ce qui est des attitudes et des valeurs, du savoir-être et du savoiragir, il est plus difficile d'agir sur ces paramètres, car il faut changer une mentalité qui est enracinée à l'échelle collective.

Pour favoriser l'esprit entrepreneurial, le Québec a décidé de mettre l'emphase sur la culture en accordant une place importante à l'entrepreneuriat, au sens des affaires et à l'argent. Cela se traduit tout d'abord par l'élimination des préjugés négatifs de la population envers l'argent et envers le modèle du chef d'entreprise en général. On tend plutôt à favoriser l'acceptation du modèle des affaires ainsi que les valeurs entrepreneuriales (autonomie, responsabilité créativité, confiance en soi, etc.). Parmi les moyens mis en œuvre pour faire naître cette culture, le Québec a choisi d'agir sur les jeunes qui ont le potentiel d'apprendre et d'enraciner les nouvelles valeurs en les sensibilisant à avoir l'entrepreneuriat comme choix de carrière désirable, mais aussi en les préparant à des compétences transversales qui leur serviront dans la prise en charge de leur vie.

\section{L'entrepreneuriat : pour une approche orientante}

La nouvelle réforme de l'éducation au Québec est en harmonie avec une approche orientante qui permet d'aider l'élève, particulièrement celui du secondaire, à anticiper et à préparer son avenir professionnel. L'école doit outiller l'élève de manière à ce qu'il puisse faire des choix éclairés qui lui permettront d'orienter son cheminement professionnel tout au long de sa vie. Au cours de son cheminement scolaire, l'élève doit êtres mis dans plusieurs contextes et pouvoir puiser à plusieurs sources d'information afin de faire face aux nouveaux défis de plus en plus nombreux sur le marché du travail.

Cette nouvelle réforme tend à rompre avec le système traditionnel de l'éducation où l'enseignant parle et l'élève écoute. Au contraire, on veut que l'élève apprenne dans l'action et que la connaissance devienne compétence, tout en favorisant l'apprentissage par projet. Les interventions éducatives devraient tendre à favoriser chez l'élève une organisation évolutive 
et constructive de sa vision au monde, de son identité et de son pouvoir d'action.

La sensibilisation à l'entrepreneuriat apparaît comme un élément important de cette approche orientante. En effet, il importe que, pendant son parcours, l'élève découvre l'entrepreneuriat comme une voie de carrière possible. Cela peut se traduire par des visites d'entreprises, des témoignages d'entrepreneurs et surtout par l'expérimentation et l'apprentissage dans l'action d'un projet entrepreneurial. Peu importe le type du projet, qu'il soit à caractère pédagogique, sportif, scientifique, technologique, communautaire ou culturel, l'essentiel est qu'à travers ce projet les élèves puissent développer des valeurs entrepreneuriales comme la confiance en soi, l'autonomie, l'esprit d'équipe, le leadership, etc.

\section{L'entrepreneuriat : un champ de compétences transversales}

Il est communément admis que la créativité, l'aptitude à la solution de problèmes, l'habileté dans les relations de travail et la capacité à s'adapter continuellement à la maîtrise des nouvelles connaissances et à l'utilisation des technologies de l'information constituent des facteurs clés de succès des individus. Cela est d'autant plus vrai dans un contexte en changement rapide. À cet effet, l'école devra poursuivre l'acquisition de compétences et d'attitudes qui ne relèvent pas du domaine exclusif de l'enseignement des disciplines. Toutes les activités éducatives organisées par l'école doivent être des occasions pour intégrer les compétences transversales. Ces dernières permettent d'actualiser le programme classique de l'éducation et de tenir compte des changements sociaux. Elles permettent de former des jeunes autonomes et responsables.

Les compétences transversales à privilégier sont regroupées en plusieurs catégories : orientation et entrepreneuriat, médias, santé et bien-être, vivre ensemble et citoyenneté, environnement et consommation. En étudiant ces thèmes à l'école, l'élève s'approprie des valeurs, des attitudes et des comportements qui lui permettront de poursuivre sa formation et de vivre en société. L'élève a l'occasion de faire des apprentissages qui font appel à plusieurs disciplines, tout en les dépassant. Le domaine général de formation « orientation et entrepreneuriat» permet d'explorer la connaissance de soi, de se familiariser avec l'approche projet et de mieux connaître les professions et les métiers. Aussi, les jeunes sont amenés à expérimenter des projets interdisciplinaires. Que les projets soient à caractère pédagogique, sportif, culturel, scientifique, artistique, technologique ou communautaire, les jeunes apprennent des habiletés entrepreneuriales dans des contextes signifiants d'apprentissage.

\section{Communiquer pour changer les attitudes}

Que cherchons-nous par le développement d'une culture entrepreneuriale? La culture entrepreneuriale n'est pas une fin en soi. En effet, le but d'une culture entrepreneuriale est de changer les mentalités vers un esprit qui encourage et favorise l'entrepreneuriat. Changer de mentalités, c'est aussi changer d'attitudes et de comportements. L'évolution des comportements constitue une dimension importante dans la réussite ou l'échec d'un changement culturel. Selon Bartoli et Hermel $^{3}$, les comportements des gens constituent des « actes conjoncturels » que nous pouvons repérer à un moment donné par observation ou par écoute. Le comportement est une variable clé du fonctionnement d'une société puisqu'il a des conséquences sur l'environnement. Selon ces mêmes auteurs, le comportement est influencé par l'environnement du moment matérialisé par un flux d'événements, par des facteurs intrinsèques aux personnalités et aux structures et comportements d'autrui dans le milieu.

\section{Il importe que, pendant son parcours, l'élève découvre I'entrepreneuriat comme une voie de carrière possible.}

Comme nous l'avons démontré dans une étude préalable ${ }^{4}$, la réussite d'un changement dépend du degré d'acceptation du changement, qui lui-même n'est autre que la traduction de la perception vis-à-vis du changement. En effet, l'individu a tendance à changer de comportement lorsqu'il a une perception positive du changement. Dans la même étude, nous avons démontré que la communication influence fortement la perception, ce qui fait qu'elle constitue un facteur clé de réussite du changement. La communication nécessite donc une attention particulière et une préoccupation de plus en plus accrue en situation de changement, quel que soit le type de ce dernier (changement culturel, structurel, technologique, etc.). 
Dans la littérature, il a été démontré que la communication autour d'un changement doit traiter quelques thèmes afin d'obtenir l'adhésion des gens. Nous présentons dans ce qui suit les thèmes qui constituent des facteurs clés de succès d'une communication du changement culturel, en faisant référence à chaque fois au cas du Québec.

\section{La nécessité du changement}

Le premier facteur clé de succès d'un message qui suscite l'adhésion au changement est la nécessité 5 . Le message d'adhésion doit apporter une réponse à la question suivante : est ce que le changement est vraiment nécessaire? Il faudrait que les gens croient que quelque chose ne va pas et doit absolument être changé (ex. : faire face au problème de la relève, le déclin démographique, etc.). Il importe donc de créer une prise de conscience de la différence entre l'état présent et l'état désiré en montrant les conséquences défavorables, les difficultés et les problèmes que rencontre la société. Pour montrer la nécessité du changement, il est utile d'expliquer ce qui a changé dans le contexte.

La nécessité permet de positionner le changement comme essentiel à la survie. Si l'on ne change pas, on sera confronté à des problèmes et à des difficultés prévisibles. En d'autres termes, le critère de nécessité fait référence à la stratégie d'évitement du pire. La nécessité répond à la question «que se passerait-il si l'on ne changeait pas? ». Ainsi, on accepte d'autant mieux un changement que notre existence est menacée par le non-changement. Par ailleurs, la nécessité peut ne pas apparaître immédiatement. Dans ce cas, les arguments la démontrant devront être pertinents. Ainsi, les arguments de nécessité sont très importants pour faire accepter le changement. Cependant, s'ils sont seuls mis en exergue, ils ne peuvent permettre l'intégration du changement.

Pour ce qui est du cas du Québec, les pouvoirs publics ont déjà préparé le terrain en sensibilisant les entrepreneurs par les forts messages et les discours à un changement culturel qui met l'accent sur le souci de la nécessité de la relève. Le changement est devenu ainsi une communication en soi. Ce message tend à influencer la culture nationale. Les journaux sont envahis de nombreux dossiers et articles portant sur la problématique de la relève, sur l'état actuel du Québec en le comparant avec les autres provinces du
Canada et avec les États-Unis. Les statistiques du GEM (Global Entrepreneurship Monitor) sont publiées en redondance, montrant que le Québec est situé en dernier lieu par rapport à ses voisins en termes d'entrepreneuriat et qu'il est primordial de rattraper ce retard. Les communications ont toutes un message disant qu'on ne peut se fier uniquement au hasard pour faire des entrepreneurs, mais qu'il faudrait donner la piqûre entrepreneuriale aux jeunes puisque ce sont eux qui prendront le flambeau de l'économie de demain. Les forums et colloques de la Fondation de l'entrepreneurship constituent aussi des lieux privilégies pour passer un tel message. Ainsi, pour ce qui est de la nécessité, premier élément de la communication d'adhésion, beaucoup d'efforts ont été apportés pour sensibiliser la population à cette problématique qui touche le Québec.

\section{La nécessité permet de positionner le changement comme essentiel à la survie.}

\section{La capacité à changer}

Plusieurs auteurs ont mentionné l'importance de communiquer des messages qui démontrent la capacité à changer ${ }^{6}$. Ainsi, le message d'adhésion doit apporter une réponse à la question suivante : pourrions-nous réussir le changement ? La communication portant sur cet élément doit augmenter la confiance dans la réussite du changement (cf. des informations sur les groupes engagés dans l'effort de changement, exemples ou modèles de réussites au sein de groupes, supports et ressources mises à disposition, formations, etc.). Le message doit montrer que les leaders s'engagent à investir du temps, de l'énergie ainsi que toutes les ressources nécessaires pour soutenir les gens dans le changement.

Au Québec, pour mettre les gens en confiance, on leur communique les ressources existant dans le milieu qui les aident à entreprendre des projets. Généralement, ce volet est assez maîtrisé par plusieurs pays sensibilisés à l'importance de l'entrepreneuriat. Il y a de plus en plus des programmes de formation sur le plan d'affaires, les techniques de ventes et de marketing, les techniques financières, etc. Ces formations se donnent aussi bien à l'université qu'au sein d'organismes d'aide destinés à ceux qui veulent se lancer en affaires. 


\section{L'intérêt}

L'intérêt représente le troisième critère influençant la perception du changement et, par le fait même, son acceptation ${ }^{7}$. En effet, un changement nécessaire et dénué d'intérêt pour les gens est souvent difficilement accepté. Le message d'adhésion doit donc apporter une réponse à la question suivante : qu'est ce que le changement va m'apporter ou nous apporter? (ex. fierté, augmentation des opportunités d'avancement et de développement...).

Là encore, les messages dans les revues et journaux québécois sont redondants, montrant le gain engendré par le changement vers une culture entrepreneuriale. Que ce soit au plan macroéconomique (amélioration de l'emploi, baisse du chômage, croissance et développement économique, etc.) ou au plan microéconomique (réalisation de soi, autonomie, adieu à la routine, etc.), l'intérêt individuel et collectif est largement mis en exergue dans les messages nationaux. Mieux encore, l'intérêt individuel est introduit non seulement par les messages dans les journaux et les discours du gouvernement, mais aussi à travers l'expérimentation de l'entrepreneuriat dès le plus jeune âge, permettant ainsi de découvrir par soi-même l'intérêt et la passion à l'entrepreneuriat, et c'est justement là la particularité du Québec.

L'apprentissage expérimental des plus jeunes à travers des projets entrepreneuriaux vise effectivement à nourrir l'intérêt à l'entrepreneuriat, à chercher la flamme entrepreneuriale endormie chez eux et à les pousser à mettre en avant leur potentiel. L'endroit idéal pour cela est le milieu scolaire: l'université mais aussi le collégial, les formations professionnelles, le secondaire et le primaire. Nous présentons dans ce qui suit des exemples de pratiques pédagogiques de sensibilisation à l'entrepreneuriat.

\section{Un changement nécessaire et dénué d'intérêt pour les gens est souvent difficilement accepté.}

\section{Pratiques pédagogiques de sensibilisation à l'entrepreneuriat}

En plus de la nouvelle réforme de l'éducation qui introduit l'entrepreneuriat comme stratégie pédagogique à l'école, le Défi de l'entrepreneuriat jeunesse vient appuyer la promotion de la culture entrepreneuriale à travers différentes mesures dont la majorité s'adresse directement aux jeunes. Ces mesures s'articulent autour de trois axes : 1) mise à contribution de l'école, 2) création d'un environnement favorable et 3) diffusion, auprès des jeunes entrepreneurs, de stratégies efficaces ${ }^{8}$.

Dans le cadre de l'axe 1 du Défi de l'entrepreneuriat jeunesse, les enseignants et les conseillers en orientation sont sensibilisés à l'importance et à la nécessité de la culture entrepreneuriale. Ainsi, ils participent de plus en plus par leur créativité à implanter des projets adaptés aux besoins de leurs étudiants et au contexte dans lequel ils opèrent. Un guide d'élaboration de projets intitulé Invitation à la culture entrepreneuriale : guide d'élaboration de projets à l'intention du personnel enseignant ${ }^{9}$ a été distribué à l'ensemble des enseignants du Québec.

De plus, le défi consolide l'action des réseaux voués à la promotion de l'entrepreneuriat auprès des jeunes. En effet, il existe plusieurs programmes entrepreneuriaux dont la démarche est préétablie et qui sont destinés aux différents niveaux d'éducation (primaire, secondaire, collégial et universitaire). Le but n'est pas de faire de tous les jeunes de futurs chefs d'entreprise, mais par l'intermédiaire de ces programmes, le Québec cherche à développer les qualités entrepreneuriales chez les jeunes, qualités nécessaires pour entreprendre sa vie, que ce soit comme chef d'entreprise, employé ou citoyen. Voici les programmes connus et réussis au Québec ainsi que des exemples concrets de projets.

\section{École micro entreprise environnementale}

Le réseau québécois des écoles micro entreprises environnementales regroupe une trentaine d'écoles primaires publiques au Québec ayant expérimenté des projets de recyclage de matériaux. Ce programme permet aux enfants du primaire d'intégrer les valeurs entrepreneuriales à travers la création d'une véritable entreprise avec un conseil d'administration et des actionnaires. Cette activité pédagogique place les élèves dans des contextes significatifs d'apprentissage et leur donne l'occasion de se faire confiance, de trouver des solutions à des problèmes et d'être davantage tenaces. De plus, ce programme sensibilise les élèves à poser des gestes favorisant la protection de l'environnement (par ex. : récupération et recyclage des re- 
buts de papier et leur transformation en produits artisanaux, fabrication du savon, plantation d'arbres, etc.). Ce sont les élèves qui déterminent la distribution des profits générés par les ventes. Ils peuvent être investis dans des activités scolaires, dans le milieu social ou dans la communauté. Les élèves accompagnés par leurs enseignants choisissent le produit qu'ils veulent vendre aux parents et à la communauté, listent les ressources nécessaires à la production, réfléchissent aux moyens de promotion et de vente et font le lancement officiel de leur entreprise ${ }^{10}$.

Prenons l'exemple d'une école micro environnementale à l'est de Montréal. Les élèves de troisième année primaire, avec le soutien de deux enseignantes, ont écrit une lettre de demande de prêt à l'attention de la directrice pour l'achat du matériel nécessaire à la production de cartes de vœux en papier recyclé Ensuite, deux élèves représentant la classe sont allés la rencontrer pour la convaincre des bienfaits de leur entreprise sur eux, l'école, l'environnement et la communauté. Comme s'ils allaient rencontrer un banquier, les élèves ont expérimenté les habiletés de communication, de vente et de négociation. La directrice a joué le jeu en posant plusieurs questions afin de les inciter à aller encore plus loin dans leur réflexion. Au final, le prêt leur a été accordé grâce à leur persévérance. Avec l'aide de leurs enseignants, ces mêmes élèves ont fait la production de cartes de voeux à partir de papier recyclé et ont organisé le lancement de leur entreprise. Des parents, des personnes de la communauté, du ministère de l'Éducation et un élu municipal ont été présents à cet événement. À l'entrée de l'école, deux élèves ont accueilli les invités en vérifiant leur nom sur la liste, certains ont animé la rencontre, d'autres ont présenté leurs produits dans des kiosques et d'autres encore se sont occupés de la caisse. «Ces activités permettent aux élèves d'intégrer les notions apprises en classe, que ce soit en mathématiques, en français ou autre », a dit une enseignante de l'école.

\section{Ce sont les élèves qui déterminent la distribution des profits générés par les ventes.}

Il est sans doute évident que mettre des jeunes élèves dans de telles situations d'apprentissage permet, en plus de les sensibiliser à l'environnement, de développer le sens des responsabilités, la confiance en soi, la créativité, les habiletés en communication et l'esprit d'équipe, tant de valeurs nécessaires pour prendre en main sa vie en tant qu'entrepreneur, intrapeneur ou citoyen en général.

\section{Jeune coop.}

Initiative du Conseil de la coopération du Québec, Jeune coop. est une activité pédagogique d'une durée de 28 semaines destinée aux élèves du secondaire et qui a pour but de les sensibiliser aux valeurs de la coopération. Les jeunes poursuivent la réalisation d'un projet collectif pour répondre à un besoin de court terme (ex. un voyage nécessitant la mise en œuvre d'activités de financement) ou de long terme (ex. besoin d'un café ou une librairie à l'école) et ce, en créant une coopérative de travail. Ils apprennent le fonctionnement de la gestion démocratique, se répartissent en comités et se réunissent en conseil d'administration pour décider de leur plan d'action et de leur échéancier ${ }^{11}$.

«Coop-Omni» est un exemple d'une jeune coop. d'une école secondaire de Trois-Rivières qui offre des services informatiques (production de documents Office, programmation de pages Web, etc.) au personel de l'école et à la communauté. Grâce à l'étude de marché qu'ils ont menée auprès du personnel de l'école, une douzaine d'élèves ont recensé le besoin et ont repéré le potentiel du service informatique. Ainsi, ils ont choisi le nom de leur coopérative et ont expérimenté sa fondation, la conception du service, la mise en marché et l'administration démocratique. Le président de «Coop-Omni », un élève de 16 ans, souhaite poursuivre ses études en informatique pour lancer sa propre entreprise d'informatique dans le futur. « Avec Coop-Omni, j'ai appris à être moins gêné avec le public et à vulgariser les termes informatiques lorsque je parle à un client. J'ai appris à me faire confiance ", dit un élève membre de la coopérative ${ }^{12}$.

\section{Entreprise étudiante}

La formule jeune entreprise existe dans plus de 100 pays. Jeune entreprise du Québec (JEQ) vise à éduquer les élèves aux valeurs de l'entreprise, à les aider à comprendre les affaires et à développer des aptitudes entrepreneuriales. JEQ offre plusieurs programmes scolaires et parascolaires (initiation aux affaires, "Les études : un choix économique », comprendre les affaires et mini entreprise ou entreprise étudiante) 
pour différents niveaux scolaires (primaire, secondaire et collégial).

Concernant l'entreprise étudiante, celle-ci est une activité scolaire ou parascolaire conçue pour offrir aux élèves de secondaire 5 et du cégep la possibilité de lancer et d'exploiter une entreprise réelle. À travers ce programme, les jeunes expérimentent le cycle complet d'une entreprise, de la création des actions jusqu'à la liquidation. Ainsi, ils apprennent à établir un plan d'affaires, à faire leur étude de marché, à fabriquer et à mettre en marché un produit ou un service, à tenir les livres comptables informatisés, à verser les salaires, à fermer les livres, à déclarer et à verser les dividendes et enfin à liquider tous les actifs et à rédiger un rapport aux actionnaires ${ }^{13}$.

« Pouffies » est un exemple d'une entreprise étudiante participant au programme Jeune entreprise. Les actionnaires sont une quinzaine d'élèves en cinquième année secondaire d'une école montréalaise. Leur entreprise produit des poufs. Ces derniers sont fabriqués à base de styromousse recyclé et dans un choix de tissus, que ce soit du polar, de la fourrure ou du satin. Les élèves eux-mêmes ont choisi le produit à vendre et ont procédé à la production et au développement des stratégies marketing. Aussi persévérant que débrouillard, le groupe a réussi à faire parler de ses produits par une animatrice d'une chaîne télévisée de musique. L'équipe n'a pas non plus eu peur du grand froid canadien lorsqu'il fallait faire du porte-àporte. Tous les membres se sont améliorés depuis le début du programme, que ce soit dans le domaine de l'organisation, de l'esprit d'équipe ou tout simplement de la rapidité de production. Sur dix élèves interviewés de cette équipe, huit ont dit qu'ils souhaiteraient avoir leur propre entreprise.

\section{Club entrepreneur étudiant (CEE)}

L'Association des clubs entrepreneurs étudiants (ACEE) du Québec regroupe plus de 70 clubs répartis au Québec. Un Club entrepreneur étudiant peut être implanté dans les centres de formation professionnelle, les cégeps et les universités. Il s'agit d'une structure qui réunit un groupe de jeunes qui veulent se familiariser avec l'entrepreneuriat et élargir leur réseau de contact afin de se préparer au marché de l'emploi ou à l'entrepreneuriat. Chaque jeune a un poste bien particulier dans la structure. Ensemble, ils décident de leur vision du club et établissent une pro-

grammation pour l'année (ex. organisation de conférences d'entrepreneurs et visites d'entreprises, jeux de l'entrepreneuriat, salon collégial des affaires, participation à un réseau d'affaires, activités bénévoles, etc. $)^{14}$.

\section{Les actionnaires sont une quinzaine d'élèves en cinquième année secondaire d'une école montréalaise.}

Comme le mentionne la présidente du club entrepreneur étudiant de l'université Laval, « le but du club est de sensibiliser les étudiants à l'entrepreneuriat et de leur permettre d'agrandir leur réseau de contacts. Par exemple, nous avons organisé un souper où, à chaque service, les étudiants changent de table, rencontrent des entrepreneurs et échangent des cartes professionnelles. Grâce à ces rencontres, des étudiants ont trouvé des associés, des mentors, des clients potentiels ou des idées d'entreprises ». Un ancien membre fondateur de ce club est aujourd'hui entrepreneur : «C'est lors des jeux de l'entrepreneuriat que j'ai rencontré mon associé. De plus, j'ai pu établir des liens avec des entrepreneurs qui sont devenus mes mentors, échanger mon expérience avec des jeunes entrepreneurs et obtenir des contrats grâce à ces contacts ${ }^{15}$.

\section{Coopérative jeunesse de service (CJS)}

Soutenue par le Regroupement québécois pour la coopération de travail, la Coopérative jeunesse de service est un outil pédagogique qui vise à promouvoir l'entrepreneuriat collectif. Une coopérative jeunesse de service est une forme de coopérative de travail réunissant des jeunes de 14 à 17 ans qui se regroupent durant la période estivale pour se créer un emploi (en offrant généralement des services de peinture, de tonte de gazon, divers travaux d'entretien, etc.). Les jeunes eux-mêmes déterminent la gamme de services qu'ils désirent offrir à la communauté, se dotent d'une structure décisionnelle coopérative et se répartissent les tâches et responsabilités afférentes à la gestion de leur coopérative. Ainsi, les jeunes bénéficient d'une formation en entrepreneuriat collectif. Ils sont soutenus et accompagnés par deux animateurs et un comité local (groupes communautaires, entreprises privés, municipalités, écoles, etc.). Généralement, le projet est initié par le comité local qui favorise l'enracinement de la CJS et facilite son implantation ${ }^{16}$. 


\section{Programme Entrepreneuriat-Études}

Ce programme, initié par le ministère de l'Éducation, combine les cours de cinquième secondaire à ceux d'une attestation de spécialisation professionnelle (ASP) dédiée à l'entrepreneuriat. Le programme joint la théorie à la pratique. En effet, les étudiants apprennent les différentes étapes du lancement d'une entreprise en l'expérimentant concrètement par la création d'une mini entreprise. Les étudiants choisissent ensemble une idée de produit ou de service et s'attribuent des postes. "J'ai découvert que j'ai un bon leadership, que je suis fort en marketing, mais que j'ai des faiblesses en finance. J'ai donc choisi d'être viceprésident finances », dit un étudiant participant au programme ${ }^{17}$. Ce programme a permis aux jeunes de mettre leur potentiel en avant et de découvrir l'entrepreneuriat comme un choix de carrière possible. Les résultats ont montré que beaucoup d'étudiants qui ont participé à ce programme veulent continuer leurs études aux niveaux collégial et universitaire dans le domaine des affaires pour ensuite lancer leur propre entreprise.

L'entrepreneur ne naît pas entrepreneur, mais ce sont son environnement (que ce soit culturel, social ou économique) ainsi que son parcours personnel et professionnel qui l'encouragent à l'entrepreneuriat.

\section{Le concours québécois en entrepreneuriat}

Le concours québécois en entrepreneuriat ${ }^{18}$ est reconnu comme le plus grand concours annuel ayant pour objectif la promotion de l'entrepreneuriat au Québec. Le concours comprend deux volets : un volet étudiant et un volet création d'entreprise. Concernant le premier volet, le concours s'adresse aux élèves et étudiants des établissements scolaires et universitaires du Québec ayant réalisé des projets permettant de développer et de mettre en valeur des habiletés entrepreneuriales. En mars 2006, ce volet a reçu 6801 projets, ce qui correspond à 164411 étudiants participants. Quant au deuxième volet, il s'adresse aux entrepreneurs ayant un projet d'entreprise et qui sont à la première étape de la réalisation. En mars 2006, ce volet a reçu 939 projets, ce qui correspond à 1461 participants. Ce concours a une grande conséquence sur la motivation et la valorisation de l'entrepreneuriat au Québec.

\section{Conclusion}

Comme le précise Fortin (2002), les entreprises créent l'emploi et la richesse d'un pays. Les entrepreneurs créent quant à eux les entreprises. L'équation est donc bien claire : il faut faire des entrepreneurs pour assurer le développement économique. C'est la raison pour laquelle le Québec a choisi d'agir sur la culture pour que l'entrepreneuriat devienne un mode de vie et que des valeurs soient intégrées et enracinées dans les habitudes des gens.

L'entrepreneur ne naît pas entrepreneur, mais ce sont son environnement (que ce soit culturel, social ou économique) ainsi que son parcours personnel et professionnel qui l'encouragent à l'entrepreneuriat. D'où l'importance de faire de cet environnement un lieu propice à la culture entrepreneuriale. Une des façons de rendre cet environnement propice à l'émergence de l'entrepreneuriat, c'est de le cultiver à l'école dès le plus jeune âge. Par ailleurs, l'enseignement traditionnel est axé plutôt sur la passivité qui est complètement à l'opposé des valeurs entrepreneuriales. Au contraire, l'apprentissage dans l'action encourage la créativité et le goût d'entreprendre. En effet, en faisant des projets entrepreneuriaux pratiques, les jeunes apprennent dans l'action et intègrent petit à petit des valeurs qui leurs serviront dans leur future carrière. Nous pensons que tous les jeunes ont un potentiel entrepreneurial, mais pour certains ce potentiel est réveillé grâce à l'exploration et à l'expérimentation, alors que pour d'autres, il est simplement endormi. Il faudrait juste le secouer par de l'apprentissage dans l'action.

L'effort de deux ans de promotion de la culture entrepreneuriale dans le cadre du défi de l'entrepreneuriat jeunesse a donné de très bons résultats. Par ailleurs, il reste beaucoup à faire! En effet, le facteur temps est crucial lorsqu'il s'agit d'un changement d'ordre culturel. Il faudrait donc poursuivre les efforts afin de réussir à changer les mentalités pour l'adoption de nouvelles attitudes.

\section{Notes et références}

Fortin, P-A. (2002), La culture entrepreneuriale, un antidote à la pauvreté, Les éditions transcontinentales Inc, Les éditions de la fondation de l'entrepreneurship, $248 \mathrm{p}$. 
2 Rendu public le 17 février 2004 par le Premier ministre Charest, ce défi est une stratégie du gouvernement québécois mise sur pied avec la participation financière du Fonds jeunesse Québec et la collaboration de nombreux partenaires du réseau québécois de l'entrepreneuriat. Cette stratégie s'est donnée trois principaux objectifs : miser sur l'école, créer un environnement favorable pour les jeunes et soutenir les jeunes entrepreneurs dans leur démarche d'entreprise (www.defi.gouv.qc.ca).

3 Bartoli, A. et P. Hermel (1986), Piloter l'entreprise en mutation: une approche stratégique du changement, Paris, Les Éditions d'Organisation.

4 Ben Ayed, H. (2004), «Changement organisationnel et dynamique des communications : le cas d'une banque tunisienne "), thèse de doctorat, sous la direction de A. Silem, Université Jean Moulin Lyon 3, France.

5 Armenakis, A.A. et S.G. Harris (2002), « Crafting a Change Message to Create Transformational Readiness », Journal of Organizational Change Management, Bradford, vol. 15, $\mathrm{n}^{\circ}$ 2, p. 169; Richardson, P. et D.K. Denton (1996), "Communicating Change », Human Resource Management, vol. 35, $\mathrm{n}^{\circ}$ 2, p 203-216.

6 Voir Armenakis et Harris, op.cit.; Bareil, C. (2004), Gérer le volet humain du changement, Les éditions de la Fondation de l'entrepreneurship.

7 Armenakis et Harris, op.cit.

8 Défi de l'entrepreneuriat jeunesse, Plan d'action triennal 2004-2005-2006, Mon avenir à ma manière : www.defi.gouv.qc.ca.
9 Pelletier, D. (2005), Invitation à la culture entrepreneuriale : guide d'élaboration de projet à l'intention du personnel enseignant, Québec, Gouvernement du Québec.

10 D'après le site Web :

http://www.entrepreneurship.qc.ca/microentreprises/

11 D'après le site Web du conseil de la coopération du Québec : http://www.coopsco.com/index.html.

12 D'après le dossier spécial entrepreneuriat: Dion, M-C. et K. Quesada (2005), «École + Entrepreneuriat = Avenir », Jobboom, janv.-fév., vol. $6, \mathrm{n}^{\circ} 1$.

13 D'après le site Web de Jeunes entreprises du Québec : http://www.jequebec.org/.

14 D'après le site Web de l'association des clubs entrepreneurs étudiant : http://www.acee.qc.ca.

15 D'après le dossier spécial entrepreneuriat: Dion, M-C. et K. Quesada (2005), op. cit.

16 D'après le site Web du Regroupement québécois pour la coopération du travail : http://www.rqct.coop/.

17 D'après le dossier spécial entrepreneuriat: Dion, M-C. et K. Quesada (2005), op. cit.

18 Concours Québécois en entrepreneuriat : http://www.concours-entrepreneur.org/. 


\section{Publicité}

MAMR 